\title{
Left ventricular systolic performance in failing heart improved acutely by left ventricular reshaping
}

Kun-Iun He, MD, PhD, a Juichiro Shimizu, MD, PhD, a Geng-hua Yi, MD, a Anguo Gu, MD, ${ }^{\text {a }}$ M. Abul Kashem, MD, PhD, ${ }^{\text {b }}$ Deborah L. Crabbe, MD, ${ }^{\mathrm{b}}$ Sulli Popilskis, DVM, ${ }^{\mathrm{c}}$ Ed X. Wu, PhD, ${ }^{\mathrm{d}}$ William P. Santamore, $\mathrm{PhD},{ }^{\mathrm{b}}$ David Melvin, MD, PhD, and Jie Wang, MD, $\mathrm{PhD}^{\mathrm{a}}$

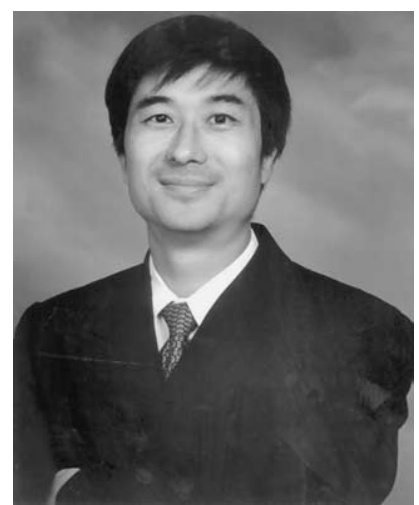

Dr Jie Wang
From the Division of Circulatory Physiology, Department of Medicine, ${ }^{\text {a }}$ Columbia University, New York, NY, Cardiology Section, ${ }^{\text {b }}$ Temple University, Philadelphia, $\mathrm{Pa}$, Department of Comparative Medicine, ${ }^{\mathrm{c}}$ Department of Radiology, ${ }^{\mathrm{d}}$ College of Physicians and Surgeons, Columbia University, New York, NY, and Division of Cardiothoracic Surgery, Department of Surgery, College of Medicine, ${ }^{\mathrm{e}}$ University of Cincinnati, Cincinnati, Ohio.

K. H., J. S., and G. H. Y. contributed equally to this work.

Supported by a research grant from CardioClasp Inc, Pine Brook, NJ.

Received for publication July 16, 2002; revisions requested Aug 13, 2002; revisions received Aug 2, 2002; accepted for publication Dec 30, 2002.

Address for reprints: Jie Wang, MD, PhD, The Heart Failure Center, Department of Medicine, Columbia University, MHB 5-435, 177 Ft Washington Ave, New York, NY 10032 (E-mail: jw147@ columbia.edu).

J Thorac Cardiovasc Surg 2003;126:56-65

Copyright (C) 2003 by The American Association for Thoracic Surgery

0022-5223/2003\$30.00+0

doi:10.1016/S0022-5223(02)73597-6
Objective: If the geometric distortion during dilated heart failure could be corrected, the tension on the myocytes would be decreased, thereby leading to an improvement in left ventricular systolic function. We tested the effects of the CardioClasp (CardioClasp Inc, Pine Brook, NJ), a left ventricular reshaping device, on the failing heart, and our empirical data were compared with computationally derived data.

Methods: Heart failure was induced by 4-week rapid cardiac pacing. At the terminal experiment, an isolated failing heart preparation (isovolumic contraction, $\mathrm{n}=5$ ) or an intact failing heart in vivo $(\mathrm{n}=7)$ was used. The effects of the reshaping device on left ventricular performance were assessed by the slopes (Ees) of the left ventricular end-systolic pressure-volume relations, hemodynamics, and echocardiograph before and after placing the CardioClasp on the heart. The change in Ees as the result of left ventricular reshaping was also estimated from computed theoretical analysis and compared with empirical data.

Results: There was a significant change in left ventricular dimension after placing the CardioClasp on the heart. In isolated heart preparation, Ees significantly increased from $1.40 \pm 0.44 \mathrm{~mm} \mathrm{Hg} / \mathrm{mL}$ to $2.42 \pm 0.63 \mathrm{~mm} \mathrm{Hg} / \mathrm{mL}$ after placing the device on the heart but returned to the baseline level $(1.46 \pm 0.27 \mathrm{~mm} \mathrm{Hg})$ after removing it. Left ventricular developed pressure and left ventricular fractional area shortening were significantly increased as the result of left ventricular reshaping. Ees derived from computed theoretical analysis was highly correlated with confirming empirical data.

Conclusions: The CardioClasp can reshape the left ventricle and improve left ventricular systolic performance in failing hearts.

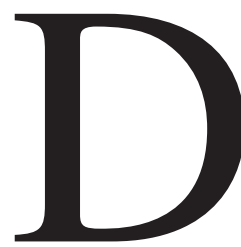

espite improved therapies to enhance survival, chronic heart failure (CHF) continues to be an unsolved dilemma and challenge for the medical community. Throughout disease progression, ventricular remodeling plays a vital role. One profound feature of remodeling is that the left ventricular (LV) chamber becomes dilated. As the LV dilates, the radius of curvature increases, placing an extra "load" on the myocytes. Consistent with Laplace's law, this results in an increase in LV wall tension and a decrease in systolic function. ${ }^{1,2}$ Pharmacologic approaches have greatly improved the management of CHF; however, the side effects of pharmacologic therapy are always of concern and unavoidable.

Surgical procedures as therapies for improving LV size and function have been reported $^{3-5}$ with controversial outcomes. Batista and colleagues ${ }^{3}$ described the partial left ventriculectomy for dilated cardiomyopathy, but many patients undergoing this procedure subsequently demonstrated ventricular arrhythmia, worsening ventricular pump function, and even death. ${ }^{6-8}$ Novel implantable devices also have been developed for the treatment of CHF. For instance, the LV assist device 


\section{A}

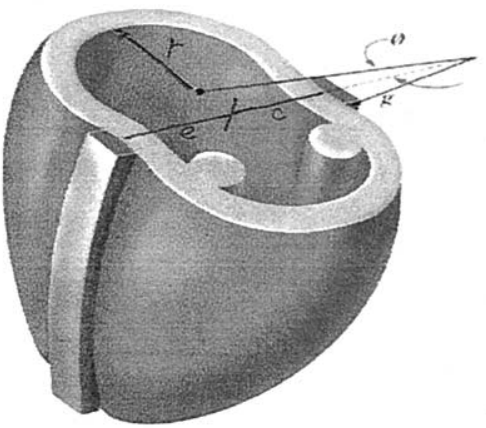

B

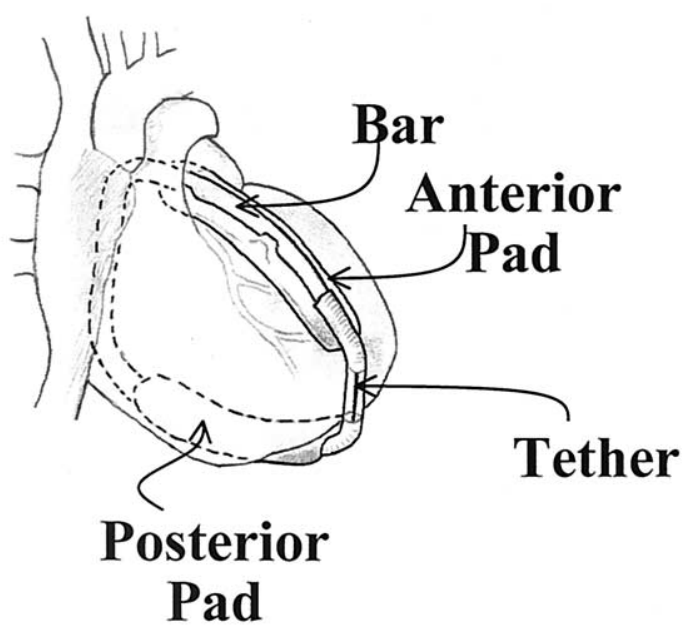

Figure 1. A, Schematic of the CardioClasp (CardioClasp Inc, Pine Brook, NJ). The device consists of 2 external structural elements to geometrically reshape the left ventricular (LV) chamber. B, Structures of the CardioClasp device and its placement on a heart.

currently acts as a life-preserving bridge for patients with end-stage heart failure until they receive a donor heart. ${ }^{9,10}$ Unfortunately, this treatment not only requires well-trained surgeons and physicians, as well as high expenses, but also carries the dangers of infection and thromboembolism.

LV reshaping exists as another option to correct the geometric burden of the dilated LV. This type of therapy rests on the hypothesis that reducing the radius of curvature directly decreases the tension on myocardial cells; thus, reducing the chamber's radius would improve systolic ventricular function. By using an LV-reshaping device, McCarthy and colleagues ${ }^{11}$ reported reduced LV wall stress and improved LV systolic function in a canine model of rapid cardiac pacing-induced heart failure. Our preliminary study also showed improved LV systolic performance after LV chamber reshaping in a failing heart. ${ }^{12,13} \mathrm{~A}$ small clinical study showed that this type of device seems to be safe. ${ }^{14}$ However, the impacts of such therapies on cardiac mechanics and their underlying physiology have not been systematically studied.

In this study, we tested the CardioClasp (CardioClasp Inc, Pine Brook, NJ), a device that uses 2 external structural elements to geometrically reshape the LV into 2 widely communicating "lobes" of reduced radius (Figure 1, A). An ex vivo isolated failing heart preparation was used in our initial study, and those empirical results were further confirmed by our observations in failing canine hearts in vivo. We developed a theoretical analysis to predict the effects of LV reshaping on a diffusely hypocontractile and dilated LV.
The correlation between the predicted and the observed alterations in LV pressure-volume relationship and the accuracy of the computational analysis, both systolic and diastolic elastance constants, were examined.

\section{Methods}

Seventeen adult mongrel dogs (7 dogs for in vivo experiments, 5 dogs for isolated heart experiments, and 5 dogs for hemodynamic support of the isolated heart experiments) weighing between 25 and $32 \mathrm{~kg}$ were used for these studies. All animals received proper and humane care in compliance with the Guide for the Care and Use of Laboratory Animals, published by the National Institutes of Health (NIH publication no. 85-23, revised 1985). This study was approved by the Institutional Animal Care and Use Committee of Columbia University.

\section{The Induction of Chronic Heart Failure}

A rapid cardiac pacing regimen resulting in $\mathrm{CHF}$ was used as described previously. ${ }^{15,16}$ The dogs were chronically instrumented for measurements of systemic hemodynamics and rapid cardiac pacing. Approximately 7 days after the surgery, a baseline hemodynamic assessment was performed while the dogs were in an awake state. Then, rapid ventricular pacing was started. The LV was paced at 210 beats/min for the first 3 weeks and then at 240 beats/min for the fourth week. After 4 weeks of the rapid cardiac pacing, measurements of the resting hemodynamics were repeated at least 40 minutes after turning off the pacer. ${ }^{16}$ The animals were divided into 2 experimental groups: (1) acute in vivo intact heart and (2) isolated heart preparation. 


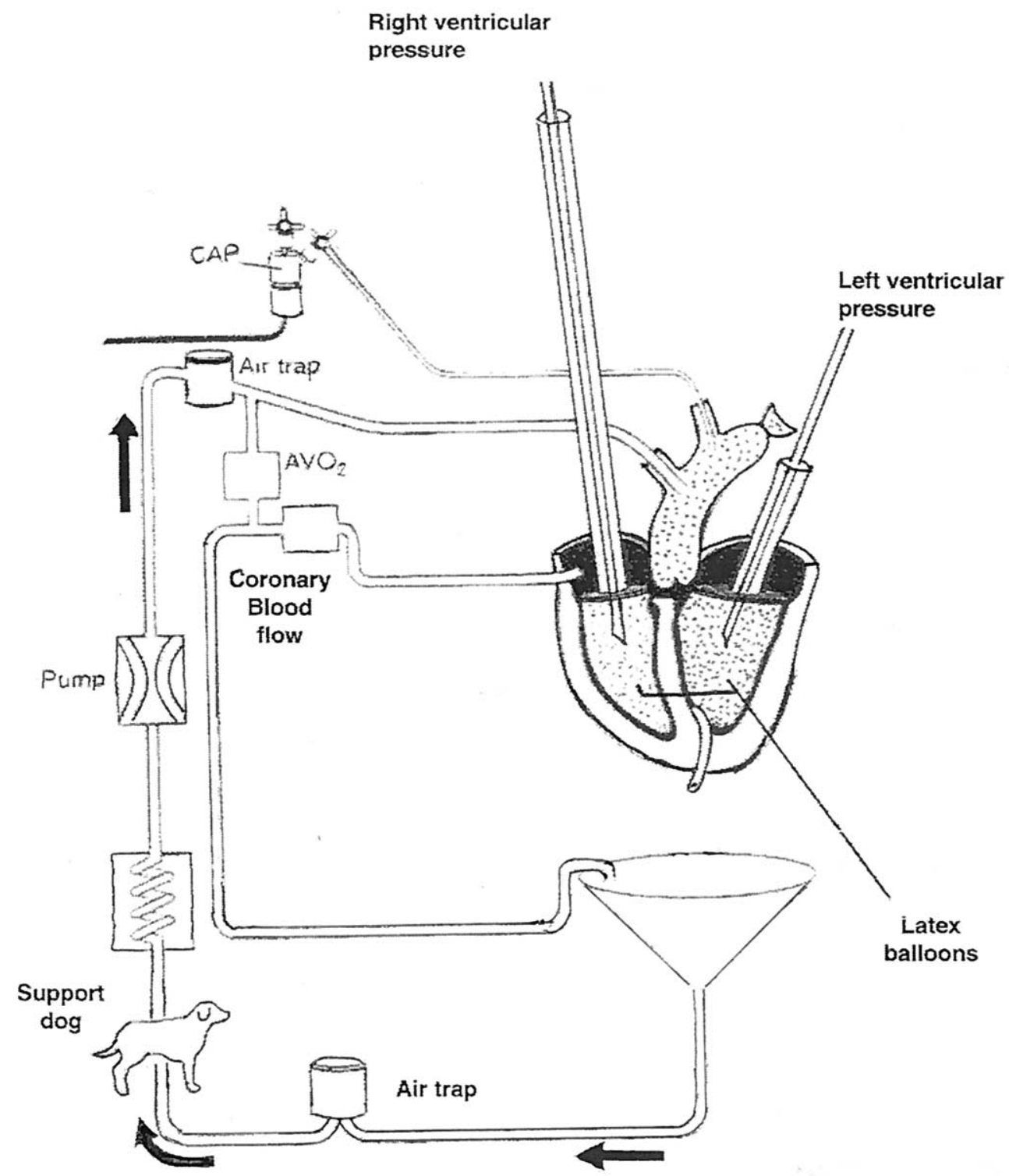

Figure 2. Schematic of isolated canine heart preparation showing donor heart perfused by blood from a "support" dog.

\section{In Vivo Preparation and Experimental Protocol}

Seven dogs were used to test the effects of the CardioClasp in vivo. Once a stable CHF state was established and baseline CHF hemodynamic assessments were made, a thoracotomy was performed at the fifth costal space to explore the heart. An ultrasonic flow probe (Transonic Systems, Ithaca, NY) was placed around the root of the ascending aorta to continuously measure cardiac output. A direct epicardial cross-sectional echocardiographic image of the LV at end-diastole was obtained. The anterior-posterior and septal-to-free wall distances were measured. The CardioClasp was placed, reducing the anterior-posterior LV end-diastolic dimension by approximately $30 \%$; then, the direct epicardial echocardiography was repeated. LV end-diastolic pressure (LVEDP) and cardiac output were also measured with the device on and off.

\section{Isolated Heart Preparation (Ex Vivo Study) and Experimental Protocol}

To test the effects of the passive LV-reshaping device, CardioClasp, on LV systolic properties, 5 isolated cross-perfused canine hearts were studied by methods similar to those described previously $^{17,18}$ and shown schematically in Figure 2. In brief, 2 dogs were anesthetized with $30 \mathrm{mg} / \mathrm{kg}$ of intravenous pentobarbital sodium. The femoral arteries and veins of 1 dog ("support dog") were cannulated and connected to a perfusion system used to supply oxygenated blood to the isolated heart. The second dog 


\section{TABLE 1. Baseline hemodynamics in awake state}

\begin{tabular}{lcc}
\hline & \multicolumn{1}{c}{ Control } & Heart failure \\
\hline LV systolic pressure $(\mathrm{mm} \mathrm{Hg})$ & $144.3 \pm 8.8$ & $112 \pm 6.0^{*}$ \\
LV end-diastolic pressure $(\mathrm{mm} \mathrm{Hg})$ & $9.1 \pm 2.0$ & $29.6 \pm 4.0^{*}$ \\
LV dP/dt (mm Hg/s) & $3192 \pm 376$ & $1578 \pm 118^{*}$ \\
MAP (mm Hg) & $107 \pm 6.2$ & $89.5 \pm 6.1^{*}$ \\
Heart rate (beats/min) & $100 \pm 12$ & $141 \pm 30^{*}$ \\
\hline
\end{tabular}

$L V$, Left ventricle; $M A P$, mean arterial pressure.

${ }^{*} P<.05$ from control.

("heart donor dog") was mechanically ventilated, and a midline sternotomy was performed. The heart was removed while being metabolically supported by the arterial flow from the support dog. The left and right atria were opened, and all the chordae tendineae were freed from the leaflets of both mitral and tricuspid valves. The isolated heart was then held by 2 metal adapters sutured to the mitral and tricuspid valve rings. These adapters connected the valve rings to a syringe system, which measured and controlled LV volume by a water-filled balloon placed inside the LV chamber. Within the balloon, a Millar pressure transducer (Millar Instruments, Inc, Houston, Texas) was placed to measure ventricular pressures. A heat exchanger maintained the perfusate temperature at $37^{\circ} \mathrm{C}$. The heart was atrially paced 10 to 15 beats/min greater than the spontaneous rate, and the coronary arterial pressure was set to approximately $100 \mathrm{~mm} \mathrm{Hg}$.

A baseline, cross-sectional echocardiographic image of the LV at end-diastole was obtained, and the anterior-posterior and septalto-free wall distances were measured. The experiments examined the effects of the CardioClasp on the end-systolic pressure-volume relationship (ESPVR) in isovolumically contracting condition. Two different interventions were performed: baseline and with the CardioClasp on the heart. At baseline, a cross-sectional echographic image of the LV was obtained at end-diastole. From this image, the anterior-posterior and septal-to-free wall distances were measured. LV pressure-volume relationships (LV loops) were obtained by continuously recording LV pressure while withdrawing fluid from the ventricular balloon once the heart was beating. A syringe pump was used for fluid withdrawal. The volume within the balloon did not change during the course of each beat (isovolumic contraction): The pressure-volume relationship for that 1 beat was a single vertical trace. The pressure was read from the intra-balloon Millar pressure transducer, and the volume was read from the known balloon content. By gradually withdrawing fluid from the balloon, a series of such straight traces can be generated. The upper ends of the traces defined the ESPVR, and the lower end of the traces presented the end-diastolic pressure-volume relationship (EDPVR). After baseline measurements were obtained, the CardioClasp device was positioned on the heart and adjusted to reduce the average anterior-posterior LV end-diastolic dimension by approximately $30 \%$. The previously mentioned echocardiographic measurements and LV pressure-volume relationship constructive procedures were repeated with the device in place. LV pressure was also recorded during a rapid removal of the CardioClasp.

\section{CardioClasp Device and Its Placement}

The CardioClasp is composed of 2 rigid bars of fixed length and curvature (Figure 1, B). These bars control the position of pivoting, flexible, epicardial contacting pads. The device, which has no blood-contacting elements, is positioned entirely around the left heart in between the pulmonary veins and behind the ascending aorta and pulmonary artery. The bars are connected by 2 flexible tethers that are adjusted to approximate the opposite walls of the LV and achieve the desired chamber radius reduction.

Variations in heart length are addressed by choice of CardioClasp size. Canine length variation has been accommodated by a choice of 3 discrete lengths. The choice of size was made by a direct measurement of the distance from the roof of the left atrium to the ventricular apex using a malleable wire measurement tool. The desired degree of reshaping was addressed for any chosen CardioClasp size by adjusting the distances between the bar ends.

A cross-sectional echocardiographic image of the LV before and after application of the device was obtained. The papillary muscles were used as internal landmarks to compare images. The distance of anterior-posterior wall (internal chamber endocardial measurement) and wall thickness were measured by using an end-diastolic image. The anterior-posterior diameter approximately corresponded to the plane in which the CardioClasp was applied. The tethers were shortened to obtain a reduction of this diameter of approximately $30 \%$.

\section{Data Collection}

From the LV pressure-volume relationship, linear regression from the points of maximal ratio between pressure and volume (top left corner of each loop) provided the slope and volume axis intercept to define the ESPVR. The slope (Ees) of the ESPVR presented the contractile function of the LV. Similarly, the EDPVR was derived by connecting the lower right corner of each loop.

Echocardiography measurements at rest were used for dimensional measurements (eg, diameter and area) of the LV during systole and diastole. LV area was determined by carefully tracing the endocardial surface and integrating the area. Mean area ejection fraction (percent) was obtained from the equation: (mean end-diastolic area - mean end-systolic area)/mean end-diastolic area, by averaging at least 5 steady-state cardiac cycles.

\section{Theoretical Analysis of Elastance Constant}

LV pressure-volume relationship can be described by the equations $\mathrm{P}_{\mathrm{es}}=\mathrm{E}_{\mathrm{es}}\left(\mathrm{V}_{\mathrm{es}}-\mathrm{V}_{\mathrm{o}}\right)$ and $\mathrm{P}_{\mathrm{ed}}=\mathrm{A}\left(\mathrm{e}^{\mathrm{k}[\mathrm{Ved}-\mathrm{Vo}]}-1\right) .\left(\mathrm{P}_{\mathrm{es}}\right.$ : end-systolic pressure; $\mathrm{P}_{\mathrm{ed}}$ : end-diastolic pressure; $\mathrm{V}_{0}$ : 0 pressure volume; $\mathrm{E}_{\mathrm{es}}, \mathrm{A}, \mathrm{K}$ : constants). On the basis of these equations, we hypothesized that intrinsic time-varying LV wall circumferential length and tension relationships held true, and that longitudinal changes were relatively minor, so the resulting new LV ESPVRs and EDPVRs could be estimated from the geometrically induced changes in the LV pressure-volume relationship. From these, we theoretically projected a new EDPVR and Ees (elastance constant and the index of LV contractile function) based on new constant values for an approximately $32 \%$ radiusreduced ventricle to match isolated heart experiments according to our hypothesis. Then we experimentally examined the constants $\mathrm{V}_{0}$, Ees, $\mathrm{A}$, and $\mathrm{K}$ by matching curves in our isolated 


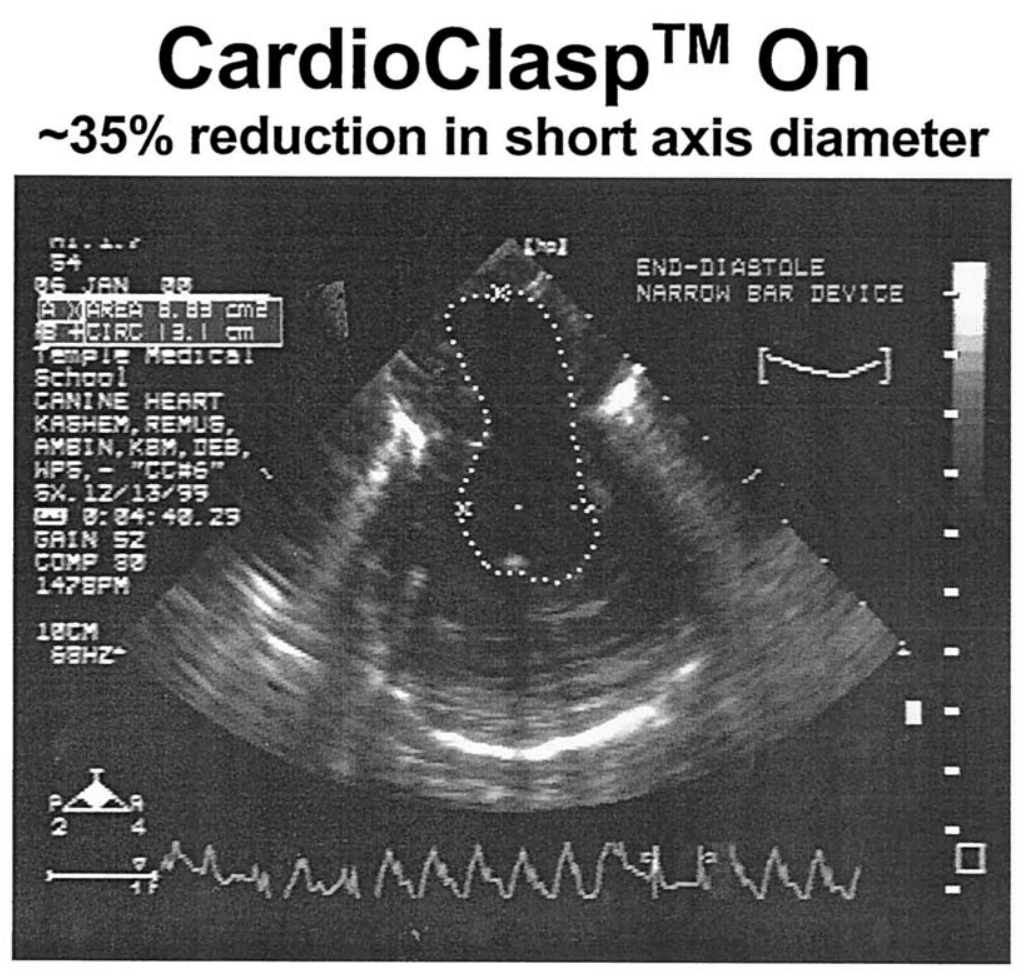

Figure 3. An echocardiograph obtained from an intact animal, indicating a significant "reshaping" of the LV once the CardioClasp was placed on the heart. In this case, there was an approximately $35 \%$ reduction in the short axis of the LV, and the bi-lobe appearance of the heart is clearly seen.

TABLE 2. Impact of CardioClasp (CardioClasp Inc., PineBrook, NJ) on left ventricle systolic and diastolic properties in vivo

\begin{tabular}{lcc}
\hline & Baseline & CardioClasp on \\
\hline LV end-diastolic area (cm) & $12.0 \pm 0.9$ & $10.4 \pm 1.3^{*}$ \\
Area ejection fraction (\%) & $0.12 \pm 0.02$ & $0.26 \pm 0.03^{*}$ \\
Cardiac output (L/min) & $2.2 \pm 0.4$ & $2.0 \pm 0.4$ \\
LVEDP (mm Hg) & $17.4 \pm 2.8$ & $16.4 \pm 2.0$
\end{tabular}

LVEDP, LV end-diastolic pressure.

${ }^{*} P<.05$ from baseline (CardioClasp off).

isovolumically contracting failing canine heart preparation $(\mathrm{n}=$ 4). The experimental curves of LV pressure-volume relationships from these hearts were generated after a $32 \% \pm 7 \%$ radius reduction by the CardioClasp. Experimental and theoretical values were compared, and the correlation between these 2 sets of data was examined to test the hypothesis underlying expectations for device-based ventricular reshaping. Detailed computational methods are described in the Appendix and have been described by Melvin. ${ }^{19}$

\section{Statistical Analysis}

The results are presented as means \pm standard deviation. Multiple linear regression analysis with Bonferroni's correction was used to test for statistical significance of differences between linear rela- tions with the CardioClasp on and off. Two-way analysis of variance was used for comparisons of hemodynamic parameters, Ees, and echocardiography parameters in different conditions.

\section{Results}

\section{In Vivo Study}

After 4 weeks of rapid cardiac pacing, severe heart failure developed, as evidenced by significantly elevated LVEDP and decreased LV dP/dt. Resting hemodynamic parameters measured in the awake state are summarized in Table 1.

Our results showed that once the CardioClasp was placed on the heart in an anesthetized state, there was a significant change in the LV dimension. Figure 3 illustrates an echocardiogram indicating the geometrical changes in LV shape. In this case, there was an approximately 35\% reduction in the short axis of the LV, and the bi-lobular appearance of the heart is clearly seen. In our group data, there was a $32 \%$ $\pm 7 \%$ reduction in LV short axis. The impacts of such reduction in $\mathrm{LV}$ dimension resulting from the placement of the CardioClasp on LV systolic and diastolic properties in vivo are shown in Table 2. LV end-diastolic area was significantly reduced, and area ejection fraction was significantly increased with the device on. Cardiac output and LVEDP were not changed. The difference in LVEDP during the heart failure state, as shown in Tables 1 and 2, was the 


\section{Device Off}

\section{Device On}

\section{$\sim 25 \%$ reduction in short axis diameter}

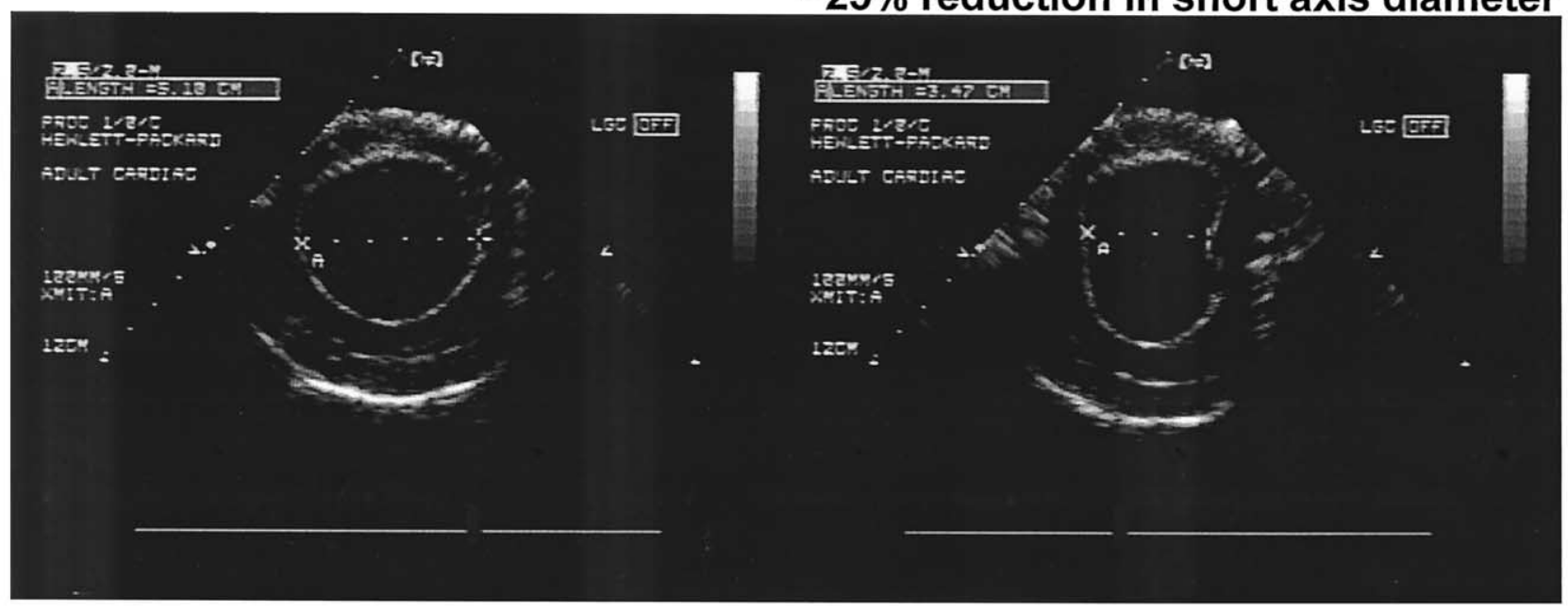

Figure 4. An echocardiograph image from an isolated heart illustrates the impact of the device on the short axis diameter and the elongation of the heart with reduction of radius of curvatures of the anterior and posterior "Iobes" of the heart.

result of measurements taken in different conditions: awake state versus anesthetized state and chest open.

\section{Isolated Heart Study}

The impacts of CardioClasp on LV performance were further examined in isolated heart preparations. Figure 4 shows an echocardiograph image from an isolated heart preparation, illustrating the impact of the device on the short axis diameter ( $\sim 25 \%$ reduction in this case) and the reduction in radii of curvature of the anterior and posterior "lobes" of the heart. There was a $32 \% \pm 7 \%$ reduction in the short axis diameter of LV. ESPVRs and EDPVRs were constructed using peak and minimum pressure during each cardiac cycle while fluid was gradually withdrawn from the intraventricular balloon. In the baseline condition (without the device), there was a linear relationship between end-systolic pressure and LV volume with a nonlinear EDPVR (Figure 5). The placement of the CardioClasp resulted in a shift toward lower volumes of both relationships. We noted that the original linear relationship of ESPVR could be accurately described by 2 linear relationships once the device was placed on the heart. Figure 5 demonstrates the changes of ESPVR and EDPVR curves resulting from the placement of the device: A "breakpoint" divided the ESPVR curve into 2 regions. Below the breakpoint volume, the ESPVR slope was basically the same as without the device, although it was shifted toward lower volume. Above the breakpoint, the ESPVR slope increased from the baseline condition, indicating a positive influence of the device on LV contractile strength. EDPVR was not significantly altered by LV reshaping. The changes in Ees (the slope of the ESPVR) are summarized in Figure 6. After CardioClasp placement, Ees significantly increased above the breakpoint, whereas Ees did not change below the breakpoint. After removing the device, Ees returned to the baseline level. The explanation for the difference of Ees above and below the breakpoint is as follows: The starling curve was defined by the relationship between LV isovolumic development pressure and end-diastolic pressure. There was no change in this relationship below a certain point because the device was simply uncoupled with the heart. The breakpoint was determined from the Starling curve using the downhill simplex algorithm $^{20}$ (see Appendix). On average, the LV volume was 57 $\pm 7 \mathrm{~mL}$, and the $\mathrm{LV}$ end-systolic pressure was $13.6 \pm 0.3$ $\mathrm{mm} \mathrm{Hg}$ at the breakpoint volume in our study. During the experiment, we observed that the device was not able to reshape the heart effectively once LV volume was below the breakpoint.

An actual LV pressure response to a rapid removal of the CardioClasp is illustrated in Figure 7. Both systolic and diastolic LV pressures decreased. However, the decrease in systolic pressure was greater than the decrease in LV diastolic pressure, and there was a slight but significant increase in LV developed pressure (LVDP), indicating that the LV generated more force once the device was placed on the heart. Table 3 summarizes the impacts of rapid removal of the CardioClasp on LVDP. 


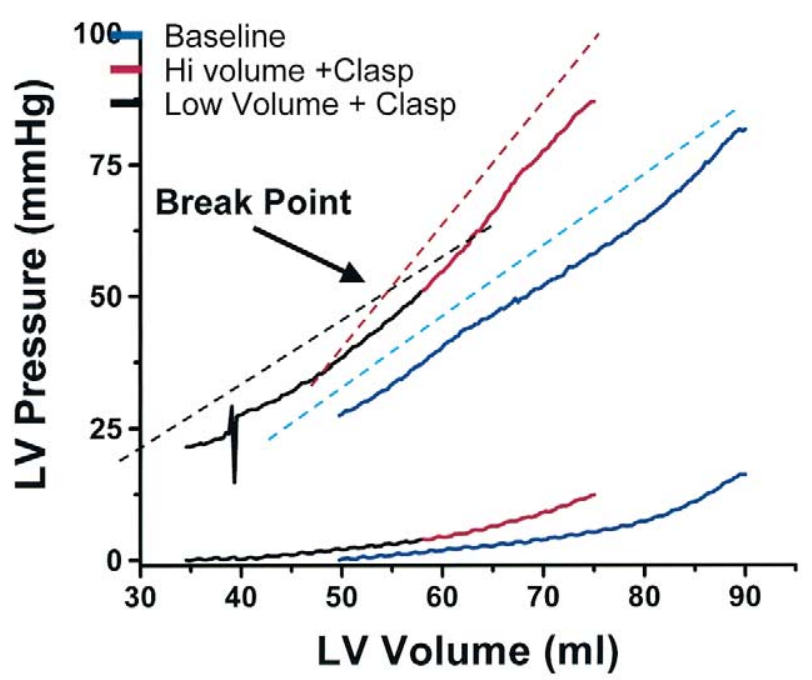

Figure 5. LV end-systolic pressure-volume relationship (ESPVR) and end-diastolic pressure-volume relationship (EDPVR) before (blue lines) and after (red and black lines) placement of the CardioClasp. Under baseline conditions (before the LV-reshaping device), there was an approximately linear relationship between LV end-systolic pressure and LV volume with a nonlinear EDPVR. CardioClasp placement resulted in a shift toward lower volumes of both relationships. It was noted that the originally linear relationship of ESPVR could be accurately described by 2 linear relationships with a breakpoint dividing the 2 regions of the curves. Below the breakpoint volume, the ESPVR slope was basically the same as under baseline conditions, although it shifted toward lower volumes. Above the breakpoint, the ESPVR slope (Ees) increased (red dot line) from baseline (blue dot line), indicating an influence of the device on LV contractile strength. EDPVR was not significantly changed by placement of the CardioClasp (CardioClasp on: + clasp).

Theoretical Analysis of Left Ventricle Reshaping Efficacy and Correlation With Empirical Data

The computed and empirical LVEDP and Ees are summarized in Table 4. The 2 sets of data represent when LV end-diastolic volume was sufficiently incrementally increased to vary the LVEDP from 12 to $20 \mathrm{~mm} \mathrm{Hg}$. There were significant increases in Ees of both theoretical and experimental observed values from baseline (shape-unaltered LV). Notably, there was a very close correlation in experimental observed and theoretical LV EDPVRs and Ees. Theoretical predictions, for both ESPVRs and EDPVRs, were grounded in the assumptions that (1) although stress-strain (or tension-length) relationships are intrinsic and not likely to acutely change, (2) both pressure-stress and strain-volume relationships are directly dependent on geometry and would change immediately (and in a quite precise, mathematically predictable fashion) with reshaping.

Observed behavior, both systolic and diastolic, was strikingly similar to that predicted. The experimental and pre-

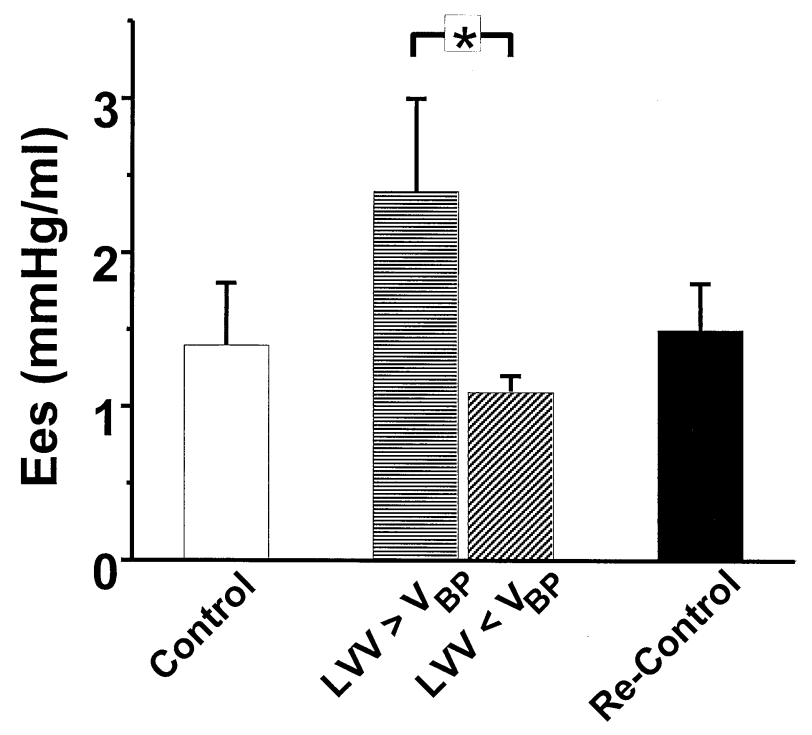

Figure 6. Ees, the slope of the ESPVR, was $1.4 \pm 0.44 \mathrm{~mm} \mathrm{Hg} / \mathrm{mL}$ at control. After CardioClasp placement, Ees increased to $2.4 \pm$ $0.63 \mathrm{~mm} \mathrm{Hg} / \mathrm{mL}$ above the breakpoint (LVV > $\mathrm{V}_{\mathrm{BP}}$ ), whereas Ees below the breakpoint ( LVV $<\mathrm{V}_{\mathrm{BP}}$ ) was not changed by CardioClasp placement. After removing the device, Ees returned to the baseline level (Re-control), $1.46 \pm 0.27 \mathrm{~mm} \mathrm{Hg} / \mathrm{mL}\left({ }^{*} P<.01\right)$.

dicted EDPVRs were almost identical, as is apparent in Table 4, with 0.97 to 0.99 coefficients of correlation in the pressure range in which there were experimental and control data. The $\mathrm{E}_{\text {es }}$ was $1.40 \mathrm{~mm} \mathrm{Hg} / \mathrm{mL}$ at baseline and was predicted from theory to increase to 2.42 with LV reshaping. It was experimentally observed to rise to a very similar value, well within the accepted error range of our instrumentation (2.35).

\section{Discussion}

The relationship between LV chamber diameter and LV wall stress can be described by Laplace's law. According to Laplace's law, when the failing heart becomes dilated, an extra load is placed on the myocardium because of the increased radius of curvature of the LV. If the radius of curvatures could be reduced, then the tension on the myocardium would be decreased (even with no change in cavity pressure), and LV systolic function could be improved. Therefore, Laplace's law has been cited as one of the major physical principles explaining the potential benefits of volume-reduction strategy. Several LV volume-reduction therapies have been introduced to clinical practice, such as the procedure of Batista and colleagues, ${ }^{3}$ the endoventricular circular patch plasty described by Dor and colleagues, ${ }^{4,5}$ and a number of other forms of ventricular aneurysmorrhaphy. ${ }^{21,22}$ However, the uses of these surgical therapies are very limited because of poor clinical benefits, such as in the Batista procedure, ${ }^{6,23,24}$ or non-applicability to the com- 


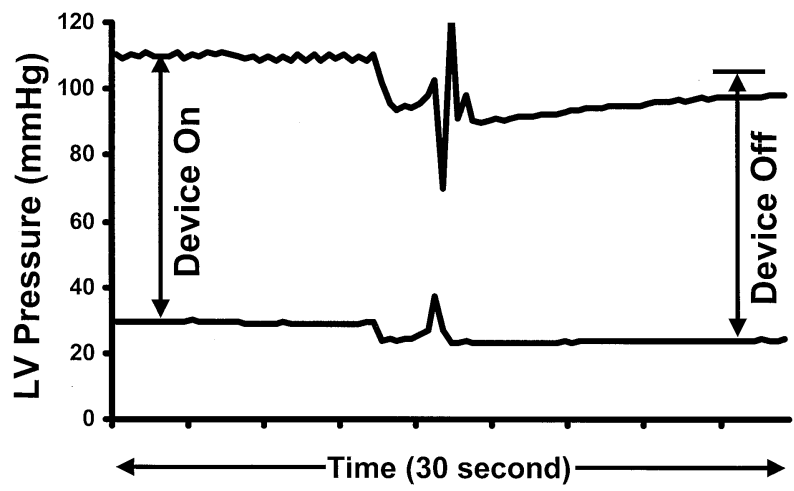

Figure 7. A typical LV pressure response to a rapid removal of the CardioClasp. Both LV systolic and diastolic pressures decreased. However, the decrease in systolic pressure was greater than the decrease in LV diastolic pressure, indicating a slight but significant increase in LV development pressure (LV development pressure: vertical line with 2 arrows).

monly occurring diffusely dilated ventricle, such as in the Dor procedure. $^{23}$

Another option is device-based remodeling of LV. By this strategy, the LV is reshaped from a single ellipsoid into 2 widely communicating ventricular "lobes," each lobe with reduced radius. Notably, the objectives of reduction in LV radius and, consequently, reduction in wall stress can be achieved in this way without sacrificing myocardium and loss of circumferential length of LV. A recent study by McCarthy and colleagues ${ }^{11}$ demonstrated that such a device improved LV ejection fraction and reduced both LV endsystolic volume and end-systolic wall stress in pacinginduced canine heart failure. Although the device used in our study is different from the one used in McCarthy and colleagues' experiment, and our device consists of 2 indenting bars (therefore reshaping the LV by the surface of the heart without entering the LV chamber), the basic working principle of these two devices is the same.

In the present study, we clearly showed the effectiveness of the device to modify the geometry of LV in both isolated heart preparation and acute in vivo experiments. The physiologic impacts of the device on the ventricular mechanical properties of failing hearts were examined in a well-documented isolated heart preparation. The load-independent and heart rate-independent index of LV contractile function, ESPVR (Ees), was used to assess any alterations of LV systolic performance once the device was placed on a failing heart, resulting in changed geometry of LV in the conditions of fixed LV volume. The ESPVR relationship was clearly shifted to the left and to a higher level by the placement of the device on a failing heart if LV volume was higher than approximately $57 \mathrm{~mL}$, correlating with endsystolic pressures of approximately 13 to $14 \mathrm{~mm} \mathrm{Hg}$. We also demonstrated a significant increase in LVDP once the
TABLE 3. Left ventricle systolic pressure and left ventricle end-diastolic pressure responses to rapid removal of the CardioClasp

\begin{tabular}{lccc}
\hline & \multicolumn{3}{c}{ LV Pressure (mm Hg) } \\
\cline { 2 - 4 } & Systolic & Diastolic & Developed \\
\hline CardioClasp off & $92 \pm 5^{*}$ & $24 \pm 2$ & $68 \pm 4$ \\
CardioClasp on & $114 \pm 6^{*}$ & $37 \pm 3^{*}$ & $77 \pm 6^{*}$ \\
Difference & $22 \pm 3$ & $13 \pm 2$ & $9 \pm 1$ \\
\hline
\end{tabular}

${ }^{*} P<.05$ from CardioClasp off.

LV reshaping device was placed on the heart. This result provided additional evidence of improved LV systolic performance as the result of the device-reshaped LV. Because all other variables were well controlled in our isolated heart preparation, the positive effects of CardioClasp on LV systolic function, indicated by a leftward shift of LV pressurevolume relationship, increased Ees, and increased LVDP, were clearly demonstrated. This conclusion was further supported by the results of echocardiography measurements from in vivo experiments. Once the CardioClasp was placed on a failing heart in vivo, area ejection fraction was significantly increased, whereas LVEDP and cardiac output were not significantly altered. This is an important observation, because it implies that if the $\mathrm{LV}$ radius is properly reshaped, beneficial effects on LV systolic function can be obtained in an intact heart. Our results are consistent with reports by Mueller and colleagues ${ }^{25,26}$ stating that hemodynamic benefits of LV reshaping were observed in bovine hearts with increased central venous pressure.

This type of correlation between theoretically predicted and experimentally observed behavior provides what is probably the only practical test of the 3 underlying assumptions supporting the hypothesis regarding the functional effects of device-based ventricular reshaping. A strain-incremental volume relationship should, of course, be measurable. Although the other 2 assumptions (effect of radius change on wall stress and the lack of an effect of that change on stress-strain relationship) may both be rather intuitive, directly confirming either would require consistent, accurate, and direct measurement of stress. No generally accepted means of performing that exists. Certainly, there may be alternative explanations for the findings, but none is readily apparent. We believe that the extremely close correlation of predicted with observed effects on both the quasi-linear end-systolic and near-exponential end-diastolic elastance curves strongly indicates that the hypothesis is indeed true. Because these predicted changes in elastance are the foundation for expected hemodynamic benefits, their experimental confirmation has substantial clinical implications.

In summary, by use of both isolated failing heart preparation and intact failing heart in vivo, we clearly demon- 
TABLE 4. Theoretic and experimental left ventricle pressure-volume relationship and Ees

\begin{tabular}{|c|c|c|c|c|c|c|c|c|c|c|}
\hline LVEDP (mm Hg) & 12 & 13 & 14 & 15 & 16 & 17 & 18 & 19 & 20 & $\begin{array}{c}\text { Ees } \\
\text { (mm Hg/mL) }\end{array}$ \\
\hline $\begin{array}{l}\text { Unaltered, } \\
\text { observed } \\
\text { LVEDV (mL) }\end{array}$ & $60.6 \pm 12.1$ & $66.0 \pm 10.9$ & $69.9 \pm 9.1$ & $73.0 \pm 8.5$ & $75.5 \pm 7.9$ & $77.0 \pm 7.6$ & $78.7 \pm 7.5$ & $80.0 \pm 7.4$ & $81.4 \pm 7.1$ & $1.40 \pm 0.44$ \\
\hline $\begin{array}{l}\text { Reshaped, } \\
\text { theoretic } \\
\text { LVEDV (mL) }\end{array}$ & $50.6 \pm 4.6$ & $52.6 \pm 4.8^{*}$ & $54.1 \pm 4.9^{*}$ & $55.6 \pm 4.7 \dagger$ & $56.7 \pm 4.8 \dagger$ & $57.6 \pm 4.8 \dagger$ & $58.2 \pm 5.1 \dagger$ & $59.5 \pm 5.2 \dagger$ & $60.7 \pm 4.9 \dagger$ & $2.42 \pm 0.63 \dagger$ \\
\hline $\begin{array}{l}\text { Reshaped, } \\
\text { observed } \\
\text { LVEDV (mL) }\end{array}$ & $44.9 \pm 5.2$ & $48.6 \pm 3.8^{*}$ & $51.8 \pm 3.8^{*}$ & $53.8 \pm 3.6^{*}$ & $55.8 \pm 3.5^{*}$ & $57.8 \pm 2.9^{*}$ & $59.5 \pm 3.3 \dagger$ & $61.0 \pm 3.2 \dagger$ & $62.4 \pm 3.6 \dagger$ & $2.35 \pm 0.74 \dagger$ \\
\hline
\end{tabular}

LVEDV, LV end-diastolic volume; Ees, slope of LV systolic pressure-volume relationship.

${ }^{*} P<.05$.

$\dagger P<.01$ from unaltered, observed LVEDS

Values are means \pm SD.

strated that the LV-reshaping device CardioClasp was able to efficiently reduce $\mathrm{LV}$ radius and remodel the geometric shape of LV chamber acutely. An immediate improved LV systolic performance was indicated by increased Ees, LVDP, and area ejection fraction once the LV reshaping device was placed on a failing heart. Computed theoretical analysis of reshaping efficacy (LV EDPVR and Ees) was highly correlated with confirming empirical data. This result further supports our conclusion of an improved LV contractile function as the result of reducing LV radius. However, further studies to confirm the long-term impact of this device on CHF and LV remodeling are warranted.

\section{References}

1. Zafeiridis A, Jeevanandam V, Houser SR, Margulies KB. Regression of cellular hypertrophy after left ventricular assist device support. Circulation. 1998;98(7):656-62.

2. Mann DL. Mechanisms and models in heart failure: a combinatorial approach. Circulation. 1999;100(9):999-1008.

3. Batista RJV, Santos JLV, Takeshita N, Bocchino L, Lima PN, Cunha MA. Partial left ventriculectomy to improve left ventricular function in end stage heart disease. J Card Surg. 1996;11:96-7.

4. Dor V, Saab M, Coste P, Kornaszewska M, Montiglio F. Left ventricular aneurysm: a new surgical approach. Thorac Cardiovasc Surg. 1989;37:11-9.

5. Dor V, Sabatier M, Montiglio F, Coste P, Di Donato M. Endoventricular patch reconstruction in large ischemic wall-motion abnormalities. J Card Surg. 1999;14:46-52.

6. McCarthy PM, Starling RC, Wong J, Scalia GM, Buda T, Vargo RL, et al. Early results with partial left ventriculectomy. $J$ Thorac Cardiovasc Surg. 1997;114:755-63.

7. Moreira LF, Stolf NA, Bocchi EA, Bacal F, Giorgi MC, Parga JR, et al. Partial left ventriculectomy with mitral valve preservation in the treatment of patients with dilated cardiomyopathy. $J$ Thorac Cardiovasc Surg. 1998;115:800-7.

8. Gorcsan J III, Feldman AM, Kormos RL, Mandarino WA, Demetris AJ, Batista RJ. Heterogeneous immediate effects of partial left ventriculectomy on cardiac performance. Circulation. 1998;97:839-42.

9. Frazier $\mathrm{OH}$. First use of an untethered, vented electric left ventricular assist device for long-term support. Circulation. 1994;89:2908-14.

10. Levin HR, Oz MC, Chen JM, Packer M, Rose EA, Burkhoff D. Reversal of chronic ventricular dilation in patients with end-stage cardiomyopathy by prolonged mechanical unloading. Circulation. 1995;91:2717-20.

11. McCarthy PM, Takagaki M, Ochiai Y, Young JB, Tabata T, Shiota T, et al. Device-based change in left ventricular shape: a new concept for the treatment of dilated cardiomyopathy. J Thorac Cardiovasc Surg. 2001;122:482-90

12. Shimizu J, Wang J, Yi GH, He KL, Kashem MA, Crabbe DL, et al. Improved systolic performance by passive remodeling in experimental heart failure. Circulation. 2000;102:II-683:3304.

13. Kashem MA, Santamore WP, Hassan S, Crabbe DL, Margulies KB, Melvin DB. CardioClasp: a new passive device to reshape cardiac enlargement. ASAIO Journal. 2002;8(3):253-9.

14. Schenk S, Reichenspurner H, Boehm DH, Groetzner J, Schirmer J, Detter C, et al. Myosplint implant and shape-change procedure: intraand peri-operative safety and feasibility. $J$ Heart Lung Transplant. 2002;21:680-6.

15. Wang J, Yi GH, Zhu SM, Gu AG, Popilskis S, Zhang H, et al. The role of angiotensin II AT1 receptor in the maintenance of hemodynamics in a canine model of coronary microembolization-induced heart failure. J Cardiovasc Pharmacol. 1999;33:335-40.

16. Wang J, Yi GH, Knecht M, Cai B, Popilskis S, Packer M, et al. Physical training alters the pathogenesis of pacing-induced heart failure through endothelium-mediated mechanisms in awake dogs. Circulation. 1997;96:2683-92.

17. Todaka K, Wang J, Yi GH, Knecht M, Stennett R, Packer M, et al. Impact of exercise training on ventricular properties in a canine model of congestive heart failure. Am J Physiol. 1997;272(3 Pt 2):H1382-90.

18. Todaka K, Wang J, Yi GH, Gu A, Zhu SM, Zhang H, et al. Effect of BAY y 5959 on myocardial function and metabolism in normal and failing hearts. Am J Physiol. 1998;274(5 Pt 2):H1560-8.

19. Melvin DB. Ventricular radius reduction without resection: a computational analysis. ASAIO J. 1999;45(3):160-5.

20. Nelder JA, Meal R. A simplex method for function minimization. Computer J. 1965;7:308-13.

21. Jatene AD. Left ventricular aneurysmectomy. Resection or reconstruction. J Thorac Cardiovasc Surg. 1985;89:321-31.

22. Cooley DA, Frazier OH, Duncan JM, Reul GJ, Krajcer Z. Intracavitary repair of ventricular aneurysm and regional dyskinesia. Ann Surg. 1992;215:417-23.

23. Artrip JH, Oz MC, Burkhoff D. Left ventricular volume reduction surgery for heart failure: a physiologic perspective. $J$ Thorac Cardiovasc Surg. 2001;122:775-82.

24. Franco-Cereceda A, McCarthy PM, Blackstone EH, Hoercher KJ, White JA, Young JB, et al. Partial left ventriculectomy for dilated cardiomyopathy: is this an alternative to transplantation? $J$ Thorac Cardiovasc Surg. 2001;121:879-93.

25. Mueller XM, Tevaearai HT, Tucker O, Boone Y, von Segesser LK. 
Reshaping the remodelled left ventricle: a new concept. Eur J Cardiothorac Surg. 2001;20:786-91.

26. Mueller XM, Tevaearai H, Boone Y, Augstburger M, von Segesser LK. An alternative to left ventricular volume reduction. J Heart Lung Transplant. 2002;21:791-6.

27. Melvin DB, Burkhoff D, Wang J. Effect of radius-reducing left ventricular reshaping on computationally predicted stroke work. J Card Surg. 2003 (in press).

\section{Appendix}

\section{Methods Used in Computational Analysis}

New values for $E_{e s}, V_{0}, A$, and $k$, describing the new pressurevolume relationships, were derived on the basis of the central hypothesis of device reshaping (ie, the time-varying circumferential tension-length relationship is unchanged, whereas pressuretension and length-volume relationships are geometrically predictable) and the assumption that the reshaping would minimally affect longitudinal behavior. For a fixed separation of reshaping members (eg, CardioClasp bars or Myosplint buttons [Myocor, Inc, Maple Grove, Minn]), the actual degree of radius reduction varies slightly through ejection and refilling. Consequently, these values are no longer truly "constants," because they will change slightly as the result of competing (although not fully canceling) effects of baseline radius reduction and wall thickening during contraction (and the reverse in relaxation).

The volume was calculated by modeling the LV cavity as 100 transverse slices. The area of each slice was computed by the addition of component areas, holding the internal circumference of that particular slice to be unchanged from the pre-reshaping state. Bar geometry was predetermined (Figure 1, A) with "g," the transverse radius of curvature being $70 \mathrm{~mm}$ and bar-width sufficient $(18 \mathrm{~mm})$ to allow tangential bar-epicardial parting and an epicardial-cavity pressure ratio no greater than 1.4 with the range of reshaping induced. Thus, the contact $\operatorname{arc} \theta$ was readily derived, and the remainder of the geometric parameters, and slice area, immediately followed. For the greatest radius reduction induced in these studies (32\%), this calculation yielded a volume reduction of approximately $12 \%$.

For example, during systole, $\mathrm{E}_{\mathrm{es}}$ describes the ESPVR. This can be considered, as can the EDPVR, to be the product of pressure-tension, tension-length, and length-volume relationships. Pressure-tension relation is proportional to the instantaneous internal radius, and length-volume is proportional to the instantaneous cross-sectional area relationship (again, assuming longitudinal contribution is negligibly affected). Thus, at any point during ejection or refilling, the new $\mathrm{E}_{\mathrm{es}}$, or " $\mathrm{E}_{\mathrm{es}}$,", will be the baseline $\mathrm{E}_{\mathrm{es}}$ divided by the product of the new and old radius ratio and the new and old area ratio.

Similarly, but with somewhat more involved mathematics, the new $\mathrm{V}_{0}$ may be shown to equal the old one times the instantaneous area ratio, the new A to be the old one divided by the instantaneous radius ratio, and the new $\mathrm{k}$ to be the old one divided by the instantaneous area ratio. Although these new values define the instantaneous pressure-volume relationships through the cardiac cycle, some of the customary intuitive definitions no longer apply. For example, it is important to note that " $\mathrm{V}_{0}$ " would now only define the volume at 0 pressure if the proportional reshaping stayed the same until the ventricle became that small. It does not: Although proportional radius reduction varies surprisingly little in physiologic pressure ranges, for most ventricles the tension members holding the reshaping bars or buttons would be slack well before 0 pressure, and the ventricle would again have a circular cross-section. In addition, although the new $\mathrm{E}_{\mathrm{es}}$ continues to define the end-systolic pressure-volume slope, that slope changes as the ventricle fills and empties, so that the ESPVR is no longer linear-it is concave upward and to the left.

These new "pseudoconstants" were computed at an arbitrary "index state" (ventricular circumference and thickness equal to that at baseline end-diastole with ventricular pressure of $25 \mathrm{~mm}$ $\mathrm{Hg}$ ) and at several hundred increments or decrements below and above that circumference both in contraction and in relaxation.

Full mathematical equations and their derivations were presented at the Fourth Symposium of the Society for Cardiac Volume Reduction, February 17, 2002 in Osaka, Japan. The manuscript of that presentation, by Drs Melvin, Burkhoff, and Wang, is under review for the Journal of Cardiac Surgery. ${ }^{27}$

\section{Calculation of the Breakpoint by Use of the Downhill Simplex Algorithm}

After placement of the CardioClasp, a breakpoint divided ESPVR into 2 regions. The downhill simplex algorithm ${ }^{20}$ was used to determine the breakpoint and ESPVRs above and below the breakpoint. The root mean squared difference between predicted lines and measured ESPVRs above and below the breakpoint can be minimized. Briefly,

A. ESPVR above the breakpoint is described as follows:

$\mathrm{LVP}=\mathrm{S}_{\text {above }} \bullet \mathrm{LVV}+\mathrm{I}_{\text {above }}(\mathrm{A})$

Where $S_{\text {above }}$ and $\mathrm{I}_{\text {above }}$ is slope and intercept of ESPVR above the breakpoint, respectively.

B. LVP at the breakpoint $\left(\operatorname{LVP}_{B}\right)$ is expressed as a function of $\mathrm{LVV}\left(\mathrm{LVV}_{\mathrm{B}}\right)$ at the breakpoint:

$\mathrm{LVP}_{\mathrm{B}}=\mathrm{S}_{\text {above }} \bullet \mathrm{LVV}_{\mathrm{B}}+\mathrm{I}_{\text {above }}(\mathrm{B})$

C. From equation (A) and (B), ESPVR below the breakpoint can be described:

$\mathrm{LVP}=\mathrm{S}_{\text {below }}\left(\mathrm{LVV}-\mathrm{LVV}_{\mathrm{B}}\right)+\mathrm{LVP}_{\mathrm{B}}\left(\mathrm{LVV}<\mathrm{LVV}_{\mathrm{B}}\right.$

Where $S_{\text {below }}$ is the slope of ESPVR below breakpoint.

Now, the optimized parameters of $\mathrm{S}_{\text {above }}, \mathrm{I}_{\text {above }}, \mathrm{S}_{\text {below }}$, and $\mathrm{LVV}_{\mathrm{B}}$ are obtained by the downhill simplex algorithm and the root mean squared difference of ESPVR above $\left(\mathrm{LVV} \geq \mathrm{LVV}_{\mathrm{B}}\right)$ and below $\left(\mathrm{LVV} \leq \mathrm{LVV}_{\mathrm{B}}\right)$ the breakpoint is minimized. 